\title{
Microdissection is essential for gene expression analysis of irradiated rectal cancer tissues
}

\author{
YASUHIRO INOUE, KOJI TANAKA, TAKESHI YOKOE, SUSUMU SAIGUSA, \\ YUJI TOIYAMA, CHIKAO MIKI and MASATO KUSUNOKI
}

\author{
Department of Gastrointestinal and Pediatric Surgery, Division of Reparative Medicine, \\ Institute of Life Sciences, Mie University Graduate School of Medicine, 2-174 Edobashi, Tsu, Mie 514-8507, Japan
}

Received March 31, 2009; Accepted May 29, 2009

DOI: $10.3892 /$ or_00000515

\begin{abstract}
Microdissection is a reliable technique and is extensively used in many cancer studies. We sought to verify the importance of the microdissection technique in molecular analysis of irradiated rectal cancer specimens. Forty patients with rectal cancer underwent 5-fluorouracil based chemoradiotherapy followed by curative surgery. We compared gene expressions that had previously been shown to be involved in chemotherapy or radiation effects; one obtained using RNA extracted from cancer cells by microdissection, and the other from bulky cancer tissues in all patients. More than 50\% regression of the primary tumor was seen in 16 patients $(40.0 \%)$. There was no significant difference in candidate gene expression profiles between tumor and stromal cells except for thymidine phosphorylase (TP). Without microdissection, there was no significant association between distant recurrence and gene expression in specimens. With microdissected sample analysis, however, patients who developed distant recurrence were found to have significantly higher intratumoral thymidylate synthase (TS), dihydropyrimidine dehydrogenase (DPD) and orotate phosphoribosyl transferase (OPRT) compared with patients without recurrence. It is possible that microdissection is essential for gene expression analysis of clinically irradiated rectal specimens because preoperative chemoradiotherapy for rectal cancer affects the tumor-stroma balance in irradiated rectal cancer specimen.
\end{abstract}

\section{Introduction}

Preoperative radiotherapy is the current standard of care for patients with locally advanced rectal cancer. However,

Correspondence to: Dr Masato Kusunoki, Department of Gastrointestinal and Pediatric Surgery, Division of Reparative Medicine, Institute of Life Sciences, Mie University Graduate School of Medicine, 2-174 Edobashi, Tsu, Mie 514-8507, Japan E-mail: kusunoki@clin.medic.mie-u.ac.jp

Key words: rectal cancer, chemoradiotherapy, microdissection the efficacy of radiotherapy is limited by significant interindividual variations in response and host toxicity. Response to preoperative radiotherapy varies depending on clinical factors such as tumor stage, radiation schedules, and concomitant chemotherapy treatment (1-3). The exact role of preoperative radiotherapy remains controversial for several reasons, especially regarding survival benefit, as distant metastasis remains a significant problem even after preoperative radiotherapy $(4,5)$. Thus current practices related to radiation technique and chemotherapy regimen differ among countries, and even among institutions within the same country. Therefore, what is an optimal schedule has not been established and adjuvant treatment for rectal cancer is a major controversy in current oncology.

To resolve this issue, increasing efforts have been directed towards developing molecular targeted therapies or in searches for molecular markers useful for predicting treatment outcome or for selecting patients for preoperative radiotherapy, based on particular tumor characteristics $(6,7)$.

Clinically resected bulky cancer tissue, however, contains not only cancers but also stromal cells, which can affect gene expression profiling and hamper accurate analysis of the cancer cells. In irradiated rectal cancer, preoperative radiotherapy affects the percentages of stroma versus tumor epithelium. These can vary widely among patients; thus this high variation further complicates comparisons of different tumor samples.

Therefore, a procedure for dissecting specific cells was needed for the clinical application of a gene expression investigation. However, no study in irradiated rectal cancer has actually compared gene expression patterns; one pattern obtained using RNA extracted from cancer cells by microdissection and the other using RNA extracted from bulky cancer tissues in the same patients. The present study investigates differences between such expression patterns; particularly looking at any association with patient survival, and discusses the importance of the microdissection technique in molecular analysis of irradiated rectal cancer specimen.

\section{Materials and methods}

Patients. A total of 40 patients with locally advanced rectal cancer were included in the current analyses. All patients were treated with preoperative chemoradiotherapy (CRT) 
followed by surgery at the Department of Gastrointestinal and Pediatric Surgery in the Mie University Graduate School of Medicine. Selection criteria were the availability and the quality of isolated RNA for real-time PCR with complete clinical data. Informed consent was obtained from each subject, and our institutional ethics committee approved the study.

CRT procedure. The tumors in all 40 patients were diagnosed as adenocarcinoma and the patients scheduled for chemoradiotherapy. Treatment was by external irradiation (10 MV photons from a linear accelerator) using a four-field box technique and patients received $20 \mathrm{~Gy}$ in 4 fractions over a period of 1 week. The irradiation field included the entire sacrum, pubic bones, and the medial portion of the ilium. Patients also underwent concurrent pharmacokinetic modulating chemotherapy (PMC; intravenous infusion of 5-FU, $750 \mathrm{mg} /$ day, and oral administration of UFT, $400 \mathrm{mg} /$ day) over a period of 1 week (8). All patients underwent curative resection with total mesorectal excision, an average of 10 days after chemoradiotherapy was completed.

Pathologic evaluation and treatment response. Pathologic evaluations of the resected specimens were performed according to TNM classifications (9). Tumor regression of the primary tumor was semiquantitatively determined by the amount of viable tumor versus the amount of fibrosis, ranging from no evidence of any treatment effect to a complete response with no viable tumor identified, as described by Dworak et al (10). Tumor regression grade (TRG) 0 was defined as no regression; TRG1, minor regression (dominant tumor with fibrosis in $\leq 25 \%$ of the tumor mass); TRG2, moderate regression (dominant tumor with fibrosis in $26-50 \%$ of the tumor mass); TRG3, good regression ( $>50 \%$ tumor regression); and TRG4, total regression (no viable tumor cells, only fibrotic mass).

RNA extraction from bulky cancer specimens. Resected specimens were frozen in liquid nitrogen at $-80^{\circ} \mathrm{C}$ until further use. Specimens were homogenized using a Mixer Mill MM 300 homogenizer (Qiagen Inc., Chatsworth, CA). Total RNA was isolated by using an RNeasy mini kit (Qiagen Inc.) according to the manufacturer's instructions.

Microdissection in formalin-fixed, paraffin-embedded (FFPE) specimens. Tumor specimens were fixed in $10 \%$ formaldehyde solution $\mathrm{v} / \mathrm{v}$ and embedded in paraffin. Sections $(10-\mu \mathrm{m})$ of FFPE specimens were stained with nuclear fast red and then manually microdissected, with reference to hematoxylin and eosin sections, to collect residual cancer and stromal cells.

RNA extraction from FFPE specimens. Microdissected samples were digested with proteinase $\mathrm{K}$ in lysis buffer containing Tris-HCl, EDTA, and sodium dodecyl sulfate as previously reported with minor modification (11). RNA was purified by phenol and chloroform extraction.

cDNA synthesis. cDNA was synthesized with a random hexamer primer and Superscript III reverse transcriptase
Table I. Primer sequences.

\begin{tabular}{ll}
\hline TS & F: 5'-GCCTCGGTGTGCCTTTCA-3' \\
& R: 5'-CCCGTGATGTGCGCAAT-3' \\
DPD & F: 5'-AGGACGCAAGGAGGGTTTG-3' \\
& R: 5'-GTCCGCCGAGTCCTTACTGA-3' \\
OPRT & F: 5'-CCAGGAGTTCAGTTGGAAGC-3' \\
& R: 5'-GGAACCTCGTTTGCCAATAA-3' \\
TP & F: 5'-GCTGGAGTCTATTCCTGGATTC-3' \\
& R: 5'-TCTGACCCACGATACAGCAG-3' \\
VEGF & F: 5'-CAGAAGGAGGAGGGCAGAA-3' \\
& R: 5'-CTCGATTGGATGGCAGTAGC-3' \\
EGFR & F: 5'-CCTATGTGCAGAGGAATTATGATCTTT-3' \\
& R: 5'-CCACTGTGTTGAGGGCAATG-3' \\
B-actin & F: 5'-ACAGAGCCTCGCCTTTGC-3' \\
& R: 5'-GCGGCGATATCATCATCC-3' \\
\hline
\end{tabular}

F, forward; R, reverse.

(Invitrogen, Carlsbad, CA) according to the manufacturer's instructions.

Real-time quantitative RT-PCR. Real-time quantitative RTPCR analysis was done using an ABI PRISM 7700 Sequence Detection System (Applied Biosystems, Inc., Foster City, $\mathrm{CA})$. Primers and probes for $\mathrm{B}$-actin, thymidine phosphorylase (TP), orotate phosphoribosyl transferase (OPRT), vascular endothelial growth factor (VEGF) and epidermal growth factor receptor (EGFR) were designed with primer3 software (Biology Workbench Version 3.2, San Diego Supercomputer Center, at the University of CA, San Diego). Primers and probes for thymidylate synthase (TS) and dihydropyrimidine dehydrogenase (DPD) were synthesized according to previously published sequences (12). Sequences are shown in Table I. PCR was done in a final volume of $25 \mu 1$ with a TaqMan Universal PCR Master Mix (Applied Biosystems) using $0.5 \mu \mathrm{l} \mathrm{cDNA}, 900 \mathrm{nM}$ of each primer, and $200 \mathrm{nM}$ of probe for the respective genes. Cycling conditions were $50^{\circ} \mathrm{C}$ for $2 \mathrm{~min}$ and $95^{\circ} \mathrm{C}$ for $10 \mathrm{~min}$ followed by 40 cycles at $95^{\circ} \mathrm{C}$ for $15 \mathrm{sec}$ and $60^{\circ} \mathrm{C}$ for $1 \mathrm{~min}$.

Relative mRNA levels of target genes. Relative mRNA levels were determined by the standard curve method. Standard curves and line equations were generated using 5-fold serially diluted solutions of cDNA from the colon cancer cell line LoVo. All standard curves were linear in the analyzed range with an acceptable correlation coefficient $\left(\mathrm{R}^{2}\right)$. The amount of target gene expression was calculated from the standard curve. Quantitative normalization of cDNA in each sample was performed using the expression of the $B$-actin gene as an internal control. Finally, mRNA levels of target genes were given as ratios to $B$-actin mRNA levels. Realtime PCR assays were done in duplicate for each sample and mean values used for calculations of the mRNA levels. 
Table II. Tumor regression grading.

\begin{tabular}{lcc}
\hline TRG & Patients no. & $\%$ \\
\hline 0 no regression & 0 & 0 \\
1 (<25\% of tumor regression) & 7 & 17.5 \\
2 (25-50\% of tumor regression) & 17 & 42.5 \\
3 ( $>50 \%$ of tumor rgression) & 16 & 40 \\
4 (complete regression) & 0 & 0 \\
\hline
\end{tabular}

TRG, tumor regression grading.

Statistical analysis. All statistical analyses were done using JMP version 5 (SAS Institute Inc. Cary, NC, USA). Values of each target gene are expressed as a median value (interquartile range). Associations between gene expression levels (continuous variables) and clinicopathological variables (categorical variables) were evaluated using Mann-Whitney U test for two groups or Kruskal-Wallis test for multiple groups. Disease-free survival was calculated from the date of surgery to the date of disease recurrence. Overall survival was calculated from the date of surgery to the date of death from rectal cancer or the last follow-up. Survival was evaluated using the Kaplan-Meier method. The log-rank test was used to compare the cumulative survival durations in the patient groups. A non-parametric receiver operating characteristic (ROC) analysis was performed to calculate the best cut-off values predictive of distant recurrence using Medcalc 7.2 for Windows (Mariakerke, Belgium). P-values $<0.05$ were considered statistically significant.

\section{Results}

Patient characteristics and survival. The age of the 40 patients ( 31 males and 9 females) ranged from 48 to 78 years (median 65). Pre-CRT clinical TNM classification revealed 2 patients with stage I (T2, N0, M0), 7 with stage II (T3-4, N0, M0), and 31 with stage III (T2-4, N1-2, M0). In particular, preCRT clinical T stages were T2 $(12.5 \%)$, T3 $(62.5 \%)$, and T4 $(25.0 \%)$. All 40 patients received preoperative chemoradiotherapy and the post-CRT pathological $\mathrm{T}$ stages were pT1 $(10 \%)$, pT2 $(25 \%)$, pT3 $(62.5 \%)$, and pT4 $(2.5 \%)$. Thirteen patients $(32.5 \%)$ had pathological lymph node metastases. Lymphatic invasion was present in 31 of the 40 patients $(77.5 \%)$, and vascular invasion in $26(65 \%)$. Thirtyfour tumors $(85 \%)$ showed well or moderately differentiated adenocarcinoma.

Among the 40 patients, 6 experienced tumor recurrence (peritoneal dissemination in 1 and lung and/or liver metastases in 5). Medians of overall survival (OS) and disease-free survival (DFS) were 38.1 months (range; 6.8-86.3) and 41.3 months (range; $2.5-86.3$ ), respectively.

Pathologic response to chemoradiotherapy. The results of TRG are summarized in Table II. TRG was as follows: TRG1, 7 patients (17.5\%); TRG2, $17(42.5 \%)$; and TRG3, $16(40 \%)$. No TRG4 (complete regression of the primary
Table III. Association between gene expression in bulky cancer tissue and distant recurrence.

\begin{tabular}{lccc}
\hline Genes & $\begin{array}{c}\text { Patients with } \\
\text { distant recurrence } \\
(\mathrm{n}=6)\end{array}$ & $\begin{array}{c}\text { Patients without } \\
\text { distant recurrence } \\
(\mathrm{n}=34)\end{array}$ & p-value \\
\hline TS & $0.65(0.17-19.83)$ & $1.33(0.82-13.23)$ & 0.88 \\
DPD & $4.21(0.70-141.25)$ & $6.52(2.30-35.64)$ & 0.94 \\
TP & $18.55(1.30-24.93)$ & $16.05(8.42-24.86)$ & 0.18 \\
OPRT & $0.46(0.005-0.38)$ & $0.37(0.26-1.08)$ & 0.35 \\
VEGF & $0.46(0.05-0.61)$ & $0.51(0.26-0.69)$ & 0.54 \\
EGFR & $0.26(0.14-0.32)$ & $0.34(0.27-0.62)$ & 0.25 \\
\hline
\end{tabular}

tumor) patients were seen in this study because there was no availability of residual cancer cells. Tumor or node downstaging was demonstrated in 23 patients $(57.5 \%)$.

\section{Molecular analysis without microdissection}

Association between our candidate gene levels in bulky cancer tissue and distant recurrence. None of the patients had local recurrence. Patterns of distant recurrence were found in 6 patients. As shown in Table III, there was no significant difference in all our candidate gene expression patterns between patients who developed distant recurrence $(n=6)$ and those without $(n=34)$.

\section{Molecular analysis with microdissection}

Comparison of gene expression levels between tumor and stromal cells. Fig. 1 shows the differences in gene expression patterns between tumor and stroma cells. TP mRNA levels were higher in tumor cells than stroma. No significant difference was seen between tumor and stroma cells in the expression of other genes (TS, DPD, OPRT, VEGF, and EGFR).

Associations between intratumoral gene expression and clinicopathological variables. Median value of post-CRT tumoral TS, DPD, TP, OPRT,VEGF, and EGFR mRNA levels on FFPE specimens were 2.32 (inter-quartile range 1.02-4.51), 5.99 (3.64-14.81), 3.99 (2.39-9.06), 0.10 (0.05$0.15), 0.032(0.018-0.040)$, and $0.013(0.009-0.020)$, respectively. Associations between TS, DPD, TP, OPRT,VEGF, and EGFR mRNA levels and clinicopathological variables are summarized in Table IV. TS and TP were significantly higher in female compared with male patients (TS; $p=0.02$, DPD; $\mathrm{p}=0.03$ ). OPRT was significantly higher in patients without vascular invasion than those with $(\mathrm{p}=0.01)$. No significant association was found between TRG and all gene expressions.

Association between our candidate gene levels and distant recurrence. Table V shows that patients who developed distant recurrence $(n=6)$ had significantly higher TS $(p=0.0104)$, DPD ( $\mathrm{p}=0.0166)$, and TP $(\mathrm{p}=0.0143)$, compared with those without recurrence $(n=34)$. No significant association between distant recurrence and OPRT was observed. Thus depending 

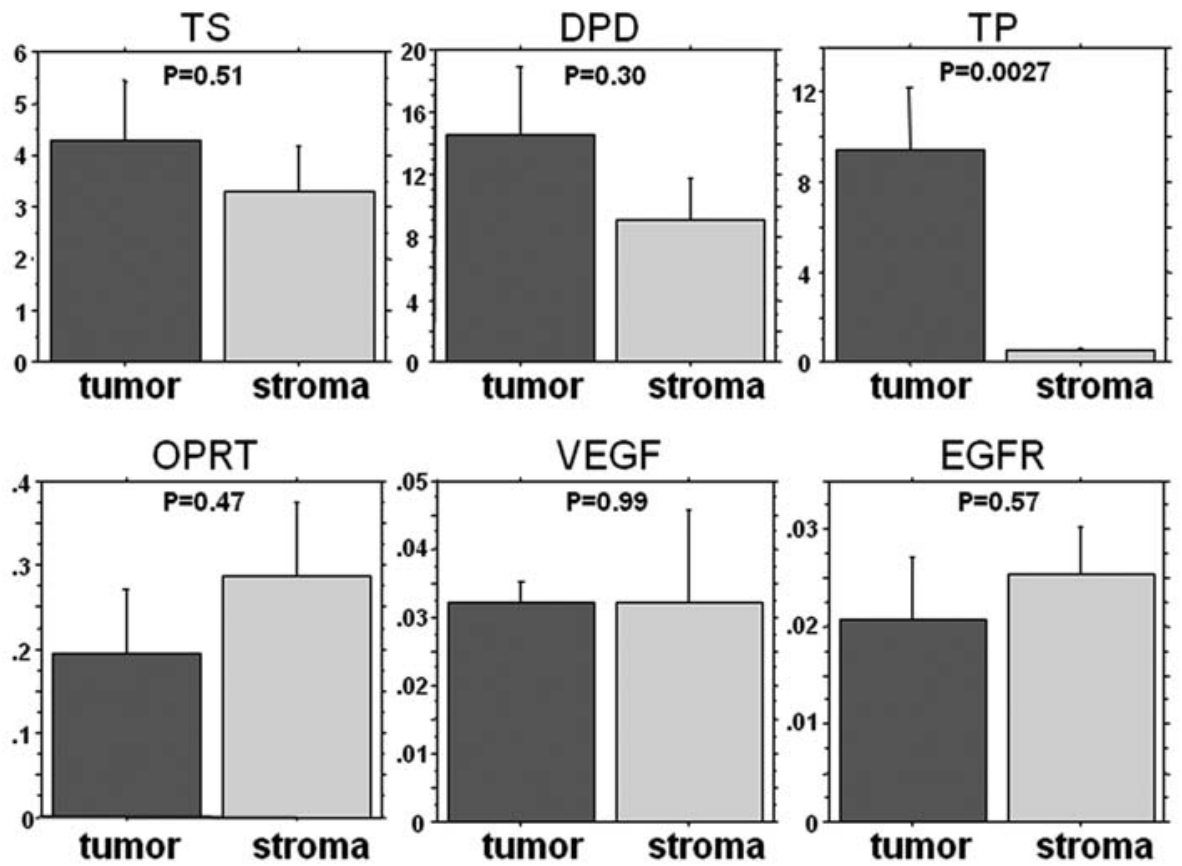

Figure 1. Differences in gene expression patterns between tumor and stromal cells.

Table IV. Association between intratumoral gene expressions and clinicopathological variables.

\begin{tabular}{|c|c|c|c|c|c|c|c|c|c|c|c|c|c|}
\hline Variable & No. & TS & p-value & DPD & p-value & $\mathrm{TP}$ & $\mathrm{p}$-value & OPRT & p-value & VEGF & p-value & EGFR & p-value \\
\hline \multicolumn{14}{|l|}{ Gender } \\
\hline Male & 31 & 1.89 & & 5.8 & & 3.84 & & 0.12 & & 0.029 & & 0.014 & \\
\hline Female & 9 & 3.53 & 0.02 & 15.88 & 0.07 & 10.15 & 0.03 & 0.05 & 0.53 & 0.038 & 0.07 & 0.01 & 0.94 \\
\hline \multicolumn{14}{|c|}{$\begin{array}{l}\text { Age } \\
\text { (median: } 65 \text { years) }\end{array}$} \\
\hline$\leq 65$ & 21 & 2.06 & & 5.8 & & 3.84 & & 0.09 & & 0.033 & & 0.014 & \\
\hline$>65$ & 19 & 2.53 & 0.28 & 6.64 & 0.34 & 4.01 & 0.42 & 0.12 & 0.95 & 0.031 & 0.75 & 0.013 & 0.36 \\
\hline \multicolumn{14}{|l|}{ Pathologic T } \\
\hline $\mathrm{T} 1$ & 4 & 1.81 & & 5.24 & & 3.43 & & 0.16 & & 0.039 & & 0.027 & \\
\hline $\mathrm{T} 2$ & 10 & 1.97 & & 6.34 & & 4.25 & & 0.09 & & 0.027 & & 0.012 & \\
\hline $\mathrm{T} 3$ & 25 & 2.71 & & 6.29 & & 4.41 & & 0.08 & & 0.031 & & 0.012 & \\
\hline $\mathrm{T} 4$ & 1 & 1.03 & 0.58 & 2.91 & 0.59 & 1.97 & 0.58 & 0.23 & 0.14 & 0.092 & 0.78 & 0.025 & 0.52 \\
\hline \multicolumn{14}{|l|}{ Pathologic N } \\
\hline Present & 13 & 2.05 & & 6.7 & & 4.01 & & 0.09 & & 0.03 & & 0.434 & \\
\hline Absent & 27 & 2.51 & 0.83 & 5.93 & 0.99 & 3.88 & 0.9 & 0.12 & 0.38 & 0.032 & 0.59 & 0.274 & 0.9 \\
\hline \multicolumn{14}{|l|}{ Histology } \\
\hline Well-moderate & 34 & 2.1 & & 5.9 & & 3.93 & & 0.11 & & 0.032 & & 0.015 & \\
\hline Poor/muc. & 6 & 3.33 & 0.34 & 12.29 & 0.29 & 9.31 & 0.29 & 0.07 & 0.52 & 0.022 & 0.12 & 0.01 & 0.17 \\
\hline \multicolumn{14}{|c|}{ Lymphatic invasion } \\
\hline Present & 31 & 2.05 & & 5.8 & & 3.88 & & 0.09 & & 0.032 & & 0.013 & \\
\hline Absent & 9 & 3.53 & 0.22 & 8.52 & 0.15 & 6.53 & 0.16 & 0.12 & 0.29 & 0.029 & 1 & 0.015 & 0.53 \\
\hline \multicolumn{14}{|l|}{ Vascular invasion } \\
\hline Present & 26 & 2.6 & & 6.17 & & 4.21 & & 0.08 & & 0.032 & & 0.013 & \\
\hline Absent & 14 & 1.58 & 0.38 & 5.9 & 0.73 & 3.71 & 0.57 & 0.13 & 0.01 & 0.031 & 0.49 & 0.015 & 0.41 \\
\hline \multicolumn{14}{|c|}{ Pathological effect } \\
\hline TRG1 & 7 & 3.06 & & 8.52 & & 5.93 & & 0.12 & & 0.025 & & 0.015 & \\
\hline TRG2 & 17 & 1.89 & & 5.65 & & 3.86 & & 0.12 & & 0.03 & & 0.014 & \\
\hline TRG3 & 16 & 2.06 & 0.64 & 6.17 & 0.56 & 4.51 & 0.66 & 0.06 & 0.22 & 0.032 & 0.96 & 0.012 & 0.64 \\
\hline
\end{tabular}


Table V. Association between gene expression in microdissected cancer tissue and distant recurrence.

\begin{tabular}{llll}
\hline Genes & \multicolumn{1}{c}{$\begin{array}{c}\text { Patients with } \\
\text { distant recurrence } \\
(\mathrm{n}=6)\end{array}$} & $\begin{array}{c}\text { Patients without } \\
\text { distant recurrence } \\
(\mathrm{n}=34)\end{array}$ & $\mathrm{p}$-value \\
\hline $\mathrm{TS}$ & $3.01(1.40-22.1)$ & $2.10(1.01-4.45)$ & 0.01 \\
$\mathrm{DPD}$ & $7.43(4.11-82.40)$ & $5.90(3.58-12.68)$ & 0.02 \\
TP & $4.97(3.12-52.25)$ & $3.92(2.31-8.04)$ & 0.01 \\
OPRT & $0.12(0.06-0.14)$ & $0.09(0.04-0.15)$ & 0.54 \\
VEGF & $0.035(0.017-0.056)$ & $0.032(0.018-0.039)$ & 0.97 \\
EGFR & $0.013(0.008-0.021)$ & $0.013(0.009-0.021)$ & 0.6 \\
\hline
\end{tabular}

on whether microdissection was used or not, we obtained different molecular analysis results.

Predictive value of TS, DPD, and TP for distant recurrence. Based on these results, receiver operating curve (ROC) analysis was used to identify each cut-off value for TS, DPD, and TP predictive of distant recurrence. Non-parametric ROC analysis showed that the best cut-off values for TS, DPD and TP were 10.26, 41.00, and 3.85, respectively. As shown in Fig. 2, patients with TS or DPD above the cut-off value showed significantly worse disease-free survival (TS, $\mathrm{p}<0.0001, \mathrm{DPD}, \mathrm{p}=0.0007)$. There was no significant difference in disease-free survival between patients with TP above the cut-off value and those below $(p=0.24)$.

\section{Discussion}

Tissue heterogeneity and the consequent need for enrichment of specific cell types before sample analysis presents a major problem in studies of cancer. The presence of contaminating cells within a sample hampers accurate molecular analysis, and the results obtained cannot easily be traced back to the biological properties of the tumor itself. Thus analysis of the molecular basis of rectal cancer involves many, often complementary techniques. In several such techniques, microdissection has been a recent development that has enabled the isolation of specific cell populations from tissue sections. The microdissection technique is reliable and is already extensively used in cancer studies to isolate specific types of cells for selected molecular analyses of DNA, RNA, and proteins.

Various studies have suggested that determining the intratumoral gene expression of important genes may be helpful for predicting clinical outcomes of patients with gastrointestinal malignancies undergoing chemotherapy or radiotherapy $(7,13)$. In the present study, we evaluated the importance of the microdissection technique for molecular analysis of irradiated rectal cancer tissue. We selected candidate genes that had previously been shown to be involved in the metabolism of 5-FU (TS, DPD, and OPRT), and in angiogenesis and radiation sensitivity (TP,VEGF, and EGFR). Previous studies in patients with colorectal cancer show that elevated intratumoral expression levels of a number of genes involved in fluoropyrimidine metabolism, including TS, DPD, and OPRT, are associated with poor response to 5-FU-based treatment (14-17). Moreover, intratumoral EGFR overexpression has been associated with resistance to preoperative radiotherapy in rectal cancer (18). The role of VEGF in angiogenesis, tumor metastasis, and clinical outcome has been demonstrated (19-21).

The microdissection technique that was extensively used in these earlier studies, where comparisons of gene expression profiles of a small number of cancer samples obtained from the microdissection were from bulky cancer tissue, indeed led to stromal cells disturbing tumor gene expression profiles (22). It has also been demonstrated that some degradation of RNA occurs during a lengthy microdissection procedure, resulting in decreased correlation between macro- and randomly microdissected samples
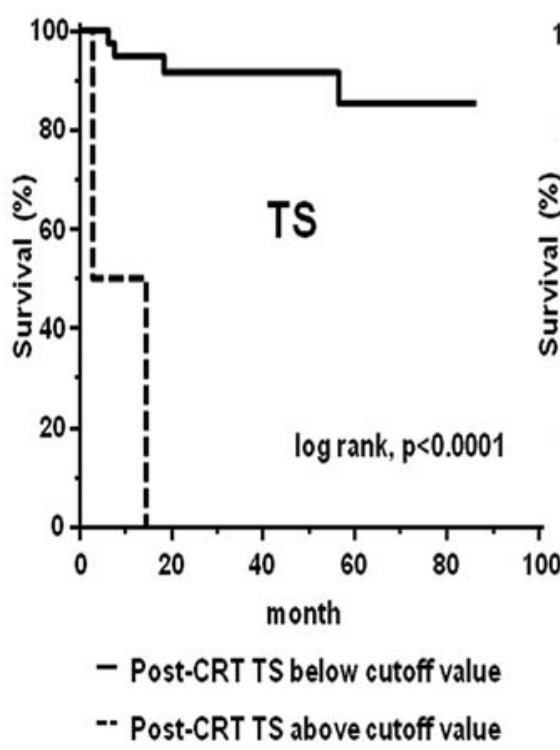
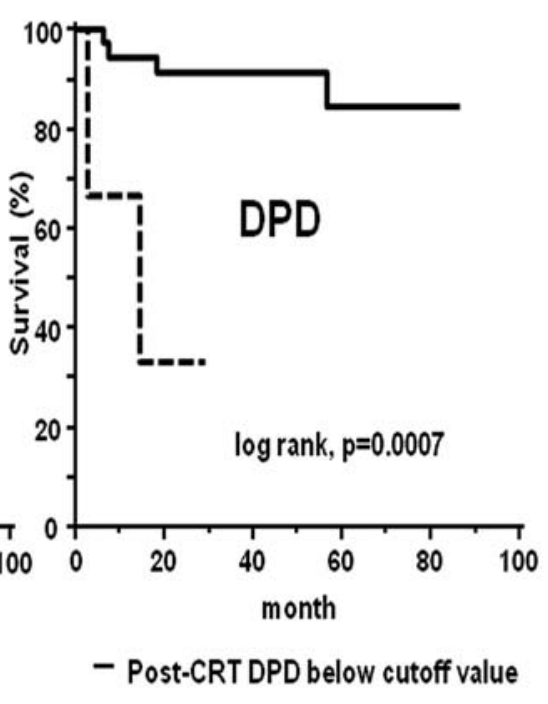

-- Post-CRT DPD above cutoff value

Figure 2. Disease-free survival according to the best cut-off value of TS or DPD. 
(23). A recent study compared gene expression profiles of several macrodissected rectal cancer samples, where only surrounding healthy tissue was removed, with the same samples microdissected by laser microdissection and concluded that the interference of stromal cells with the overall gene expression profiles appeared to be minor (24). Thus the more convenient macrodissection procedure can be adequate for the identification of tumor cell-specific gene expression profiles. However, when high percentages of stroma are present in the cancer samples, the influence of stromal cells might prevent accurate analysis of gene expressions specific for epithelial tumor cells. Especially, recent advances in chemoradiotherapy for rectal cancer reveal remarkable pathological effects in irradiated cancer samples (25). Indeed, tumor downstaging after preoperative chemoradiation therapy occurs in $46.7 \%$, including $17.9 \%$ with complete responses (26). Our study showed $57.5 \%$ pathological tumor or lymph node downstaging. Therefore, a high percentage of tumor cells were replaced by stroma after preoperative chemoradiotherapy. It follows that manual microdissection is slow, cumbersome, and easily subject to contamination compared with laser microdissection. We confirmed the difference in intratumoral gene expression analysis between samples presented with or without manual microdissection.

In conclusion, because preoperative chemoradiotherapy for rectal cancer affects the tumor-stroma balance in irradiated rectal cancer specimens, microdissection is essential for gene expression analysis of clinically irradiated rectal specimens.

\section{References}

1. Díaz-González JA, Calvo FA, Cortés J, et al: Prognostic factors for disease-free survival in patients with T3-4 or N+ rectal cancer treated with preoperative chemoradiation therapy, surgery, and intraoperative irradiation. Int J Radiat Oncol Biol Phys 64: 1122-1128, 2006.

2. Benzoni E, Intersimone D, Terrosu G, et al: Prognostic value of tumour regression grading and depth of neoplastic infiltration within the perirectal fat after combined neoadjuvant chemoradiotherapy and surgery for rectal cancer. J Clin Pathol 59: 505-512, 2006.

3. Braendengen M, Tveit KM, Berglund A, et al: Randomized phase III study comparing preoperative radiotherapy with chemoradiotherapy in non-resectable rectal cancer. J Clin Oncol 26: 3687-3694, 2008 .

4. Trial SRC: Improved survival with preoperative radiotherapy in resectable rectal cancer. Swedish Rectal Cancer Trial. N Engl J Med 336: 980-987, 1997.

5. Ahmad NR and Nagle D: Long-term results of preoperative radiation therapy alone for stage $\mathrm{T} 3$ and $\mathrm{T} 4$ rectal cancer. $\mathrm{Br}$ J Surg 84: 1445-1448, 1997.

6. Geng L, Donnelly E, McMahon G, et al : Inhibition of vascular endothelial growth factor receptor signaling leads to reversal of tumor resistance to radiotherapy. Cancer Res 61: 2413-2419, 2001 .

7. Schneider S, Park DJ, Yang D, et al: Gene expression in tumor-adjacent normal tissue is associated with recurrence in patients with rectal cancer treated with adjuvant chemoradiation. Pharmacogenet Genomics 16: 555-563, 2006.

8. Sobin LH and Fleming ID: TNM Classification of Malignant Tumors. 5th edition (1997). Union Internationale Contre le Cancer and the American Joint Committee on Cancer. Cancer 80: 1803-1804, 1997.
9. Dworak O, Keilholz L and Hoffmann A: Pathological features of rectal cancer after preoperative radiochemotherapy. Int J Colorectal Dis 12: 19-23, 1997.

10. Kusunoki M, Yanagi H, Noda M and Yamamura T: The usefulness of pharmacokinetic modulating chemotherapy (UFT plus $5 \mathrm{FU}$ ) in the treatment of unresectable colorectal carcinomas. Oncol Rep 6: 547-552, 1999.

11. Bijwaard KE, Aguilera NS, Monczak Y, et al: Quantitative realtime reverse transcription-PCR assay for cyclin D1 expression: utility in the diagnosis of mantle cell lymphoma. Clin Chem 47: 195-201, 2001 .

12. Schneider S, Uchida K, Brabender J, et al: Downregulation of TS, DPD, ERCC1, GST-Pi, EGFR, and HER2 gene expression after neoadjuvant three-modality treatment in patients with esophageal cancer. J Am Coll Surg 200: 336-344, 2005.

13. Kang SY, Han JH, Lee KJ, et al: Low expression of Bax predicts poor prognosis in patients with locally advanced esophageal cancer treated with definitive chemoradiotherapy. Clin Cancer Res 13: 4146-4153, 2007.

14. Salonga D, Danenberg KD, Johnson M, et al: Colorectal tumors responding to 5-fluorouracil have low gene expression levels of dihydropyrimidine dehydrogenase, thymidylate synthase, and thymidine phosphorylase. Clin Cancer Res 6: 1322-1327, 2000.

15. Leichman CG, Lenz HJ, Leichman L, et al: Quantitation of intratumoral thymidylate synthase expression predicts for disseminated colorectal cancer response and resistance to protracted-infusion fluorouracil and weekly leucovorin. J Clin Oncol 15: 3223-3229, 1997.

16. Ichikawa W, Uetake H, Shirota Y, et al: Combination of dihydropyrimidine dehydrogenase and thymidylate synthase gene expressions in primary tumors as predictive parameters for the efficacy of fluoropyrimidine-based chemotherapy for metastatic colorectal cancer. Clin Cancer Res 9: 786-791, 2003.

17. Fujii R, Seshimo A and Kameoka S: Relationships between the expression of thymidylate synthase, dihydropyrimidine dehydrogenase, and orotate phosphoribosyltransferase and cell proliferative activity and 5-fluorouracil sensitivity in colorectal carcinoma. Int J Clin Oncol 8: 72-78, 2003.

18. Giralt J, Eraso A, Armengol M, et al: Epidermal growth factor receptor is a predictor of tumor response in locally advanced rectal cancer patients treated with preoperative radiotherapy. Int J Radiat Oncol Biol Phys 54: 1460-1465, 2002.

19. Ishigami SI, Arii S, Furutani M, et al: Predictive value of vascular endothelial growth factor (VEGF) in metastasis and prognosis of human colorectal cancer. Br J Cancer 78: 1379-1384, 1998.

20. Takahashi T, Shirasawa T, Miyake K, et al: Protein tyrosine kinases expressed in glomeruli and cultured glomerular cells: Flt-1 and VEGF expression in renal mesangial cells. Biochem Biophys Res Commun 209: 218-226, 1995.

21. Gupta VK, Jaskowiak NT, Beckett MA, et al: Vascular endothelial growth factor enhances endothelial cell survival and tumor radioresistance. Cancer J 8: 47-54, 2002.

22. Sugiyama Y, Sugiyama K, Hirai Y, et al: Microdissection is essential for gene expression profiling of clinically resected cancer tissues. Am J Clin Pathol 117: 109-116, 2002.

23. Michel C, Desdouets C, Sacre-Salem B, et al: Liver gene expression profiles of rats treated with clofibric acid: comparison of whole liver and laser capture microdissected liver. Am J Pathol 163: 2191-2199, 2003.

24. De Bruin EC, van de Pas S, Lips EH, et al: Macrodissection versus microdissection of rectal carcinoma: minor influence of stroma cells to tumor cell gene expression profiles. BMC Genomics 6: 142, 2005.

25. Glynne-Jones R, Wallace M, Livingstone JI and MeyrickThomas J: Complete clinical response after preoperative chemoradiation in rectal cancer: is a 'wait and see' policy justified? Dis Colon Rectum 51: 10-19, 2008.

26. Gavioli M, Luppi G, Losi L, et al: Incidence and clinical impact of sterilized disease and minimal residual disease after preoperative radiochemotherapy for rectal cancer. Dis Colon Rectum 48: 1851-1857, 2005. 